\title{
Wave-current interaction with a vertical square cylinder at different Reynolds numbers
}

\author{
Azhen Kang • Bing Zhu
}

Received: 5 June 2012/Revised: 10 September 2012/ Accepted: 25 September 2012/Published online: 5 June 2013

(C) The Author(s) 2013. This article is published with open access at Springerlink.com

\begin{abstract}
Large eddy simulation is performed to study three-dimensional wave-current interaction with a square cylinder at different Reynolds numbers, ranging from 1,000 to 600,000 . The Keulegan-Carpenter number is relevantly a constant of 0.6 for all cases. The Strouhal number, the mean and the RMS values of the effective drag coefficient in the streamwise and transverse directions are computed for various Reynolds numbers, and the velocity of a representative point in the turbulent zone is simulated to find the turbulent feature. It is found that the wave-current interaction should be considered as three-dimensional flow when the Reynolds number is high; under wave-current effect, there exists a critical Reynolds number, and when the Reynolds number is smaller than the critical one, current effect on wave can be nearly neglected; conversely, with the Reynolds number increasing, wave-currentstructure interaction is sensitive to the Reynolds number.
\end{abstract}

Keywords Large eddy simulation (LES) .

Wave-current-structure interaction - Drag coefficient .

Vortex shedding $\cdot$ Reynolds number

\section{Introduction}

In recent years, the highway and road systems have gone through a rapid expansion in China, resulting in the construction of many sea bridges. The piers of these bridges deep into sea must endure large wave forces and tidal actions. The high ocean waves and turbulent currents often

\footnotetext{
A. Kang $(\bowtie) \cdot$ B. Zhu

School of Civil Engineering, Southwest Jiaotong University, Chengdu 610031, China

e-mail: xiaokang_198610@163.com
}

cause the large vibration or deformation of bridges. The design for sea bridge piers relies on the accurate prediction of wave-current forces and vortex-shedding frequency. Accordingly, wave-current-structure interaction is a focus in the studies of coastal and offshore bridges.

Park et al. [1] used the linear potential theory to investigate the fully nonlinear wave-current-body interaction in terms of three-dimensional numerical tank. His study was based on weak current and no flow separation. However, in reality, flow separation will always occur even though the current is weak. Thus, in order to deal with the physical problems of wave-current-structure interactions, the turbulence closure model was developed. Deardorff [2] established a large eddy simulation (LES) model which solves the large-scale eddy motions and modeled the smallscale turbulent fluctuations to solve the turbulent flows with large Reynolds numbers. Sohankar [3] applied a LES model to studying flow interaction with a bluff body from moderate-to-high Reynolds numbers by employing two different sub-grid scale models, namely, the Smagorinsky and a dynamic one-equation models. Koo and Kim [4] investigated nonlinear wave-current interactions with fixed or freely floating bodies using a two-dimensional fullynonlinear numerical wave tank (NWT). Li and Lin [5] developed a two-dimensional numerical tank to simulate the coaction processes based on the Reynolds-averaged Navier-Stokes equations. Cheng et al. [6] used the lattice Boltzmann method to simulate a two-dimensional incompressible linear shear flow over a square cylinder and investigated the effect of shear rate on the frequency of vortex shedding as well as the drag force. The complexities of flow and turbulence patterns as well as the pressure fields induced by the wave-current attacks require a threedimensional model to predict the deformation of piers of bridge structures. Vengadesan and Nakayama [7] evaluated 
turbulent flow over a square cylinder by employing three subgrid-scale SGS stress closure LES models. Lin and Li [8-11] used LES to study wave-current-body interaction, and obtained lots of significant revelations of nonlinear wave-current-body interactions. Tan [12,13] applied an LES model to simulate three-dimensional wave interaction with structures and studied wave-current-body interactions.

Based on the Navier-Stokes equation, the wave-generation method of defining inlet boundary conditions is applied in this article to build an LES model. The model is then used to study wave-current interaction with a vertical square cylinder for various Reynolds numbers. The drag force and vortex feature caused by the nonlinear wavecurrent-structure interaction are, respectively, numerically simulated with various high Reynolds numbers. The mean, RMS of drag coefficient and the Strouhal number which represent the vortex shedding frequency are calculated and compared.

\section{Theoretical model}

\subsection{Model description}

The governing equations for spatially averaged mean flow are as follows, which are obtained by filtering the classical Navier-Stokes equations [2]:

$$
\begin{aligned}
& \frac{\overline{\partial u_{i}}}{\partial x_{i}}=0, \\
& \frac{\partial \overline{u_{i}}}{\partial t}+\frac{\partial}{\partial x_{j}}\left(\overline{u_{i} u_{j}}\right)=-\frac{1}{\rho} \frac{\partial \bar{p}}{\partial x_{i}}+\overline{g_{i}}+\frac{1}{\rho} \frac{\partial \bar{\tau}_{i j}}{\partial x_{j}},
\end{aligned}
$$

where $i=j=1,2,3$ represent the three directions of threedimensional fluid particle, the variables with overbars are spatially averaged quantities, $\rho$ is the fluid density, $g_{i}$ is the gravitational acceleration in the $i$ th component, $\bar{u}_{i}$ is the mean velocity in the $i$ th component, $\bar{p}$ is the filtered pressure, and $\bar{\tau}_{i j}$ is the viscous stress of filtered velocity field. The subgrid scale tensors are defined by the difference of $\overline{u_{i} u_{j}}$ and $\overline{u_{i} u_{j}}$ produced from the filtering, which can be described by means of sub-grid Reynolds stress:

$\overline{\tau_{i j}^{R}}=-\rho\left(\overline{u_{i} u_{j}}-\overline{u_{i} u_{j}}\right)$.

The sub-grid model is solved based on the eddy viscosity model hypothesis; thus, the isotropic residual stress tensor is defined as follows:

$\overline{\tau_{i j}^{r}}=\overline{\tau_{i j}^{R}}-\frac{2}{3} k_{r} \delta_{i j}$,

where $k_{r}=\frac{1}{2} \tau_{i j}^{R}$ is residual kinetic energy, and $\delta_{i j}$ is Kronecker delta function. From Eq. (4), the isotropic residual stress tensor terms can be absorbed into the filtered pressure terms, i.e.,

$\bar{P}=\bar{p}+\frac{2}{3} k_{r}$

By Substituting Eqs. (3)-(5) into Eq. (2), we transform the filtered momentum equation into

$\frac{\partial \overline{u_{i}}}{\partial t}+\frac{\partial}{\partial x_{j}}\left(\overline{u_{i} u_{j}}\right)=-\frac{1}{\rho} \frac{\partial \bar{P}}{\partial x_{i}}+\overline{g_{i}}+\frac{1}{\rho} \frac{\partial \bar{\tau}_{i j}}{\partial x_{j}}+\frac{1}{\rho} \frac{\partial \overline{\tau_{i j}^{r}}}{\partial x_{j}}$.

The Smagorinsky sub-grid scale model [14] is applied to calculate $\overline{\tau_{i j}^{r}}$, that is,

$\overline{\tau_{i j}^{r}}=2 \rho \gamma_{t} \bar{S}_{i j}=\rho \gamma_{t}\left(\frac{\partial \bar{u}_{i}}{\partial x_{j}}+\frac{\partial \bar{u}_{j}}{\partial x_{i}}\right)$

where eddy viscosity coefficient $\gamma_{t}=L_{s}^{2} \sqrt{2 \bar{S}_{i j} \bar{S}_{i j}}$, Smagorinsky length scale $L_{s}=C_{s} W$, in which $C_{s}$ is the Smagorinsky model dimensionless coefficients ranging from 0.1 to 0.2 due to various calculating fluids. In this study, $C_{s}$ is taken to be $0.15, W$ is the length scale of minimum vortex, and $W=\left(\Delta_{x}+\Delta_{y}+\Delta_{z}\right)^{1 / 3}$, where $\Delta_{x}, \Delta_{y}, \Delta_{z}$ are the grid spacings of $x, y$, and $z$ directions. Thus, the LES momentum equations which are obtained by means of Smagorinsky sub-grid model without the filtered signs can be described as

$$
\begin{aligned}
\frac{\partial u_{i}}{\partial t}+\frac{\partial}{\partial x_{j}}\left(u_{i} u_{j}\right)= & -\frac{1}{\rho} \frac{\partial P}{\partial x_{i}}+g_{i} \\
& +\frac{1}{\rho} \frac{\partial}{\partial x_{j}}\left[\rho\left(\gamma+\gamma_{t}\right)\left(\frac{\partial \bar{u}_{i}}{\partial x_{j}}+\frac{\partial \bar{u}_{j}}{\partial x_{i}}\right)\right], \\
\frac{\partial u_{i}}{\partial x_{i}}=0, &
\end{aligned}
$$

where $\gamma$ is the molecular viscous coefficient.

To trace the three-dimensional free surface transformation, the so-called $\sigma$-coordinate transformation [15] is applied to map the irregular physical domain into a cube where the free surface and bottom boundary condition can be set precisely. In this study, operator-splitting method [16] is used to solve Eqs. (8) and (9).

\subsection{Model validation}

To validate the numerical model mentioned above, a threedimensional model is set up. The square cylinder is vertically located in a numerical wave-current basin with the dimension of $30 \mathrm{~m} \times 10 \mathrm{~m} \times 1 \mathrm{~m}$. The still water depth is $1 \mathrm{~m}$. The side length of square cylinder is $1 \mathrm{~m} \times 1 \mathrm{~m}$, and its height is $1 \mathrm{~m}$. The center of the cylinder is located at the centerline in the $y$ direction and $10 \mathrm{~m}$ away from the left boundary. A uniform and undisturbed current with the speed of $0.22 \mathrm{~m} / \mathrm{s}$ is set on the inflow boundary conditions; 
the corresponding Reynolds number is $R e=u_{0} \times L / v=$ $2.2 \times 10^{4}$. A nonuniform mesh system is used on the horizontal plan with the grids of $130 \times 80$. Near the square cylinder, the finest grids $\Delta x=\Delta y=0.005 \mathrm{~m}$ is deployed and coarser grids father away. In the vertical direction, the uniform grids are used, 20 grids in total. A timestep of $\Delta t=0.001 \mathrm{~s}$ is used in the computation. The computation time $t$ is a dimensionless value by parameter $l / u_{0}$, and force is a dimensionless value by parameter $\rho u_{0}^{2}$. Totally 200 dimensionless $t$ are calculated.

The time history curves of drag and lift coefficient are given in Fig. 1. The mean force coefficients $\left(C_{d}\right)$, meansquare deviation of drag and lift coefficients $\left(C_{d}^{\prime}\right.$ and $\left.C_{l}^{\prime}\right)$, and normalized shedding frequencies (Strouhal number $\mathrm{St}$ ) are given in Table 1 .

The data in Table 1 are analyzed from $100 t$ to $200 t$ (where $t=t^{*} u_{0} / l$ ) during which the turbulence has

(a)

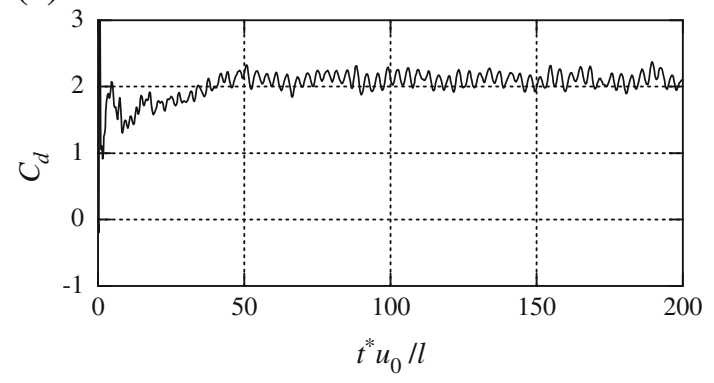

(b)

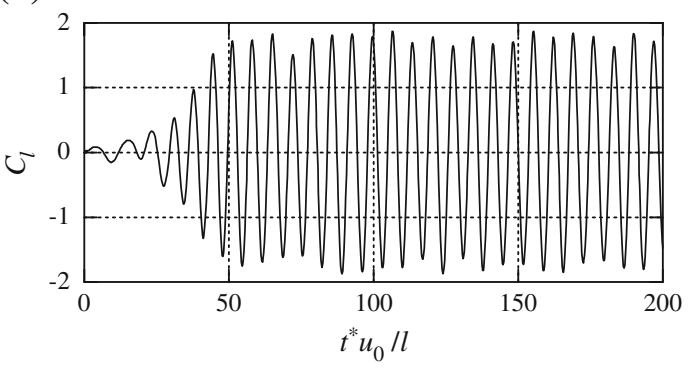

Fig. 1 The time history of drag and lift coefficients. a $C_{d}$, b $C_{l}$

Table 1 Calculated mean force coefficient $\left(C_{d}\right)$, mean-square deviation of drag and lift coefficients $\left(C_{l}^{\prime}\right.$ and $\left.C_{d}^{\prime}\right)$, and normalized shedding frequencies (Strouhal number St)

\begin{tabular}{lllll}
\hline Item & $C_{l}^{\prime}$ & $C_{d}$ & $C_{d}^{\prime}$ & $\mathrm{St}$ \\
\hline This article & 1.2475 & 2.1094 & 0.1892 & 0.139 \\
Ref. [17], $R e=22,000$ & - & $2.05-2.23$ & & 0.135 \\
Vickery, 1 [18] & 1.32 & - & 0.17 & 0.12 \\
Vickery, 2 [18] & 1.27 & - & 0.17 & - \\
Lee, $R e=176,000[19]$ & 1.22 & 2.05 & 0.22 & - \\
\hline
\end{tabular}

achieved full development. The corresponding numerical results are compared with Lyn's experiment results [17]. It can be seen from the comparison that the numerical result agrees with the experiment result well.

\subsection{Model conditions}

In this study, the three-dimensional wave-current interaction with a vertical square cylinder at various Reynolds numbers is investigated. The numerical model is the same as that of Sect. 2.2 (see Fig. 2a). Inflow boundary condition is generally set on the left side of computational domain where both free surface and velocities are provided based on either laboratory measurements or theoretical expression. Thus, a linear wave train with a wave period of $4 \mathrm{~s}$ and a wave height of $0.05 \mathrm{~m}$ together with the current is sent from the left boundary. The corresponding Reynolds number ranges from $1.0 \times 10^{4}$ to $6.0 \times 10^{5}$ and the current speeds from 0.001 to $0.6 \mathrm{~m} / \mathrm{s}$. The value of $K C=u_{p} T / L \approx 0.6$. The right side of computational domain is set by radiation boundary that can absorb the wave and flow energy. On free surface, the zero normal and tangential stresses are enforced with zero pressure. On bottom or solid wall boundary, no-slip boundary is applied. A nonuniform mesh system is used on the horizontal plan with the grids of $130 \times 80$. Near the square cylinder, the finest grids $\Delta x=\Delta y=0.05 \mathrm{~m}$ are deployed and coarser grids father away. In the vertical direction, the uniform grids are used, 20 grids in total.

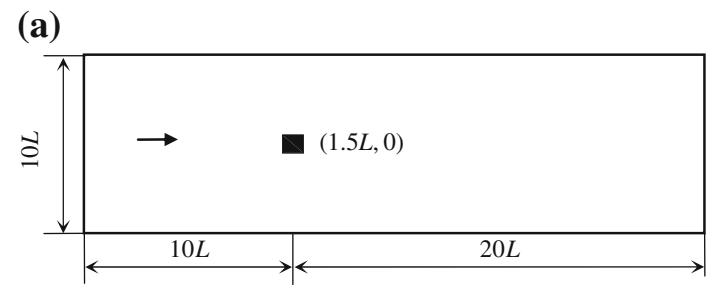

(b)

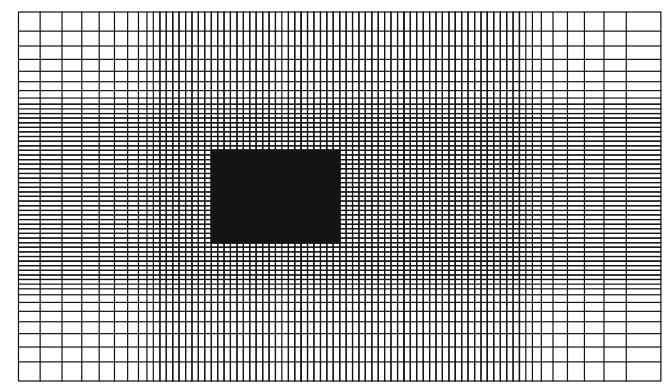

Fig. 2 Mesh arrangement near the square cylinder on the horizontal plane. a Computational domain arrangement, b Mesh arrangement near the square cylinder 


\section{Results and discussions}

In Fig. 3, the calculated vorticity on the middle elevation at the same time moment $t=100 \mathrm{~s}$ for different Reynolds numbers is plotted as a gray scale color map. Two extreme cases of wave-only case $(K C=0.6)$ and current-only case $\left(R e=6.0 \times 10^{5}\right)$ are also discussed.

Figure $3 \mathrm{a}-\mathrm{c}$ show that the current is so weak that its effect on wave can be neglected when $R e$ is less than $1.5 \times 10^{5}$. The flow is essentially symmetric about the
Fig. 3 Calculated vorticity on the middle elevation at $t=100 \mathrm{~s}$ for different Reynolds numbers. a Wave only, b $K C=0.6, R e=10,000$ c $K C=0.6, R e=100,000$, d $K C=0.6, R e=150,000$, e $K C=0.6, R e=200,000$, f $K C=0.6, R e=300,000$, g $K C=0.6, R e=400,000$, h $K C=0.6, R e=500,000$, i $K C=0.6, R e=600,000$, j $K C=0, \operatorname{Re}=600,000$
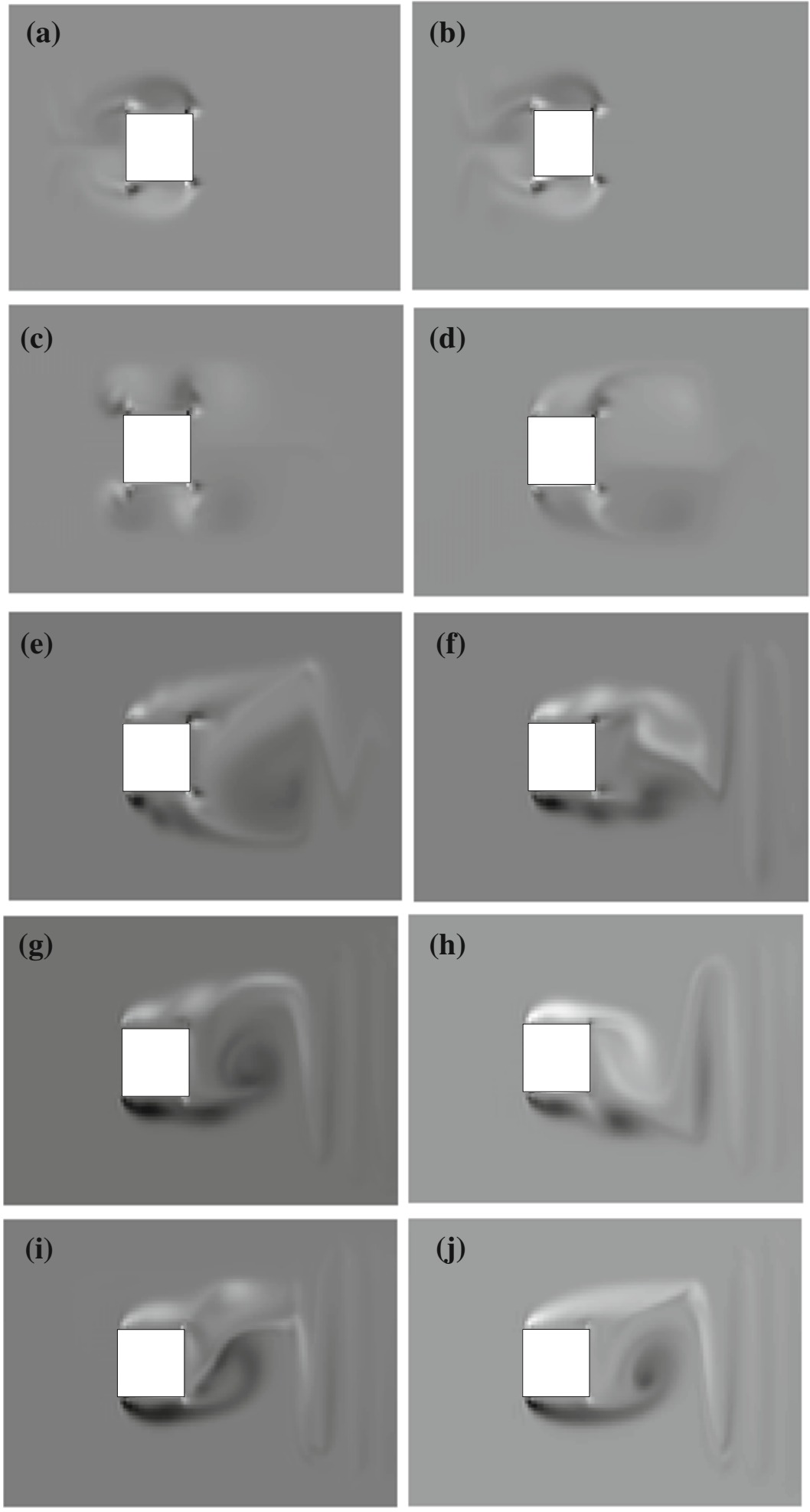
centerline in the $y$ direction during all time history under constant $K C$ number and relatively weak current effect. Flow separations from corners exist but the vortices are attached to the structure. Under wave-only effect of wave height $H=0.05 \mathrm{~m}$ as well as wave period $T=4 \mathrm{~s}$, the value of $K C=u_{p} T / L \approx 0.6$, where $u_{p}$ is the maximum wave-induced fluid particle velocity, $u_{p} \approx 0.15 \mathrm{~m} / \mathrm{s}$ [10]. Thus, when the Reynolds number magnitude is smaller (a)

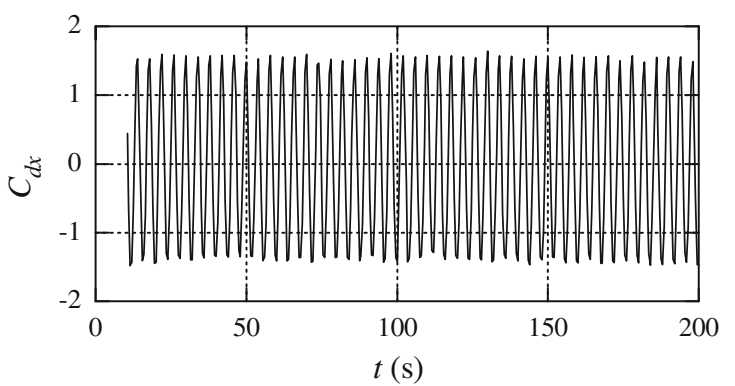

(c)

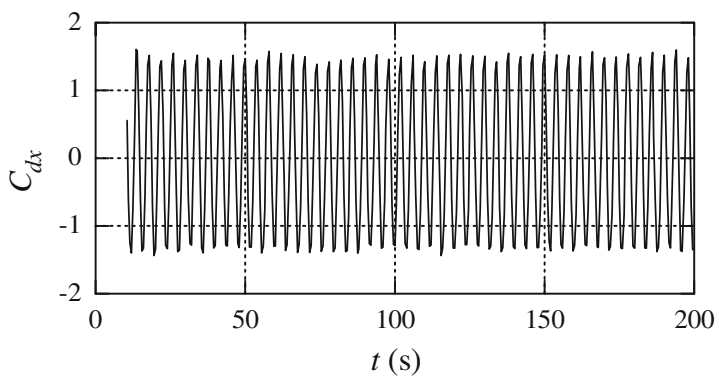

(e)

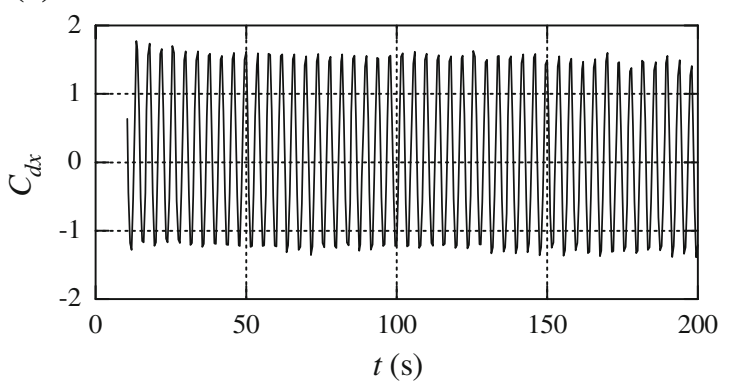

(g)

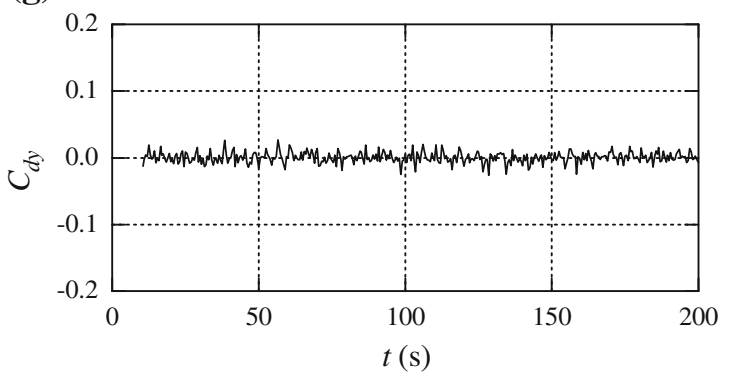

(b)

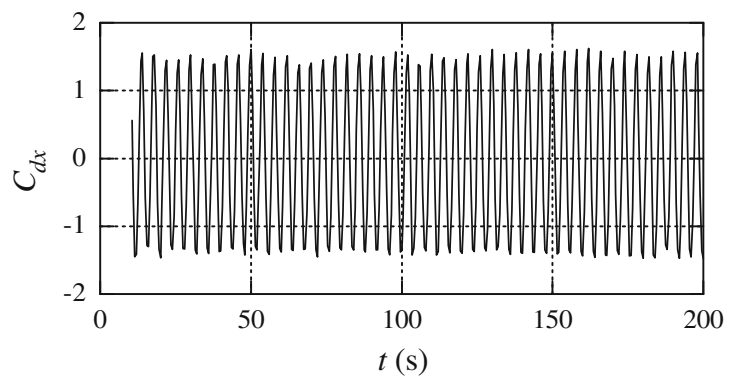

(d)

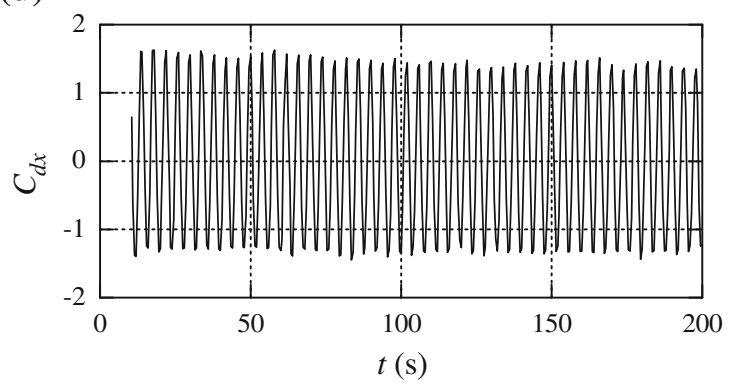

(f)

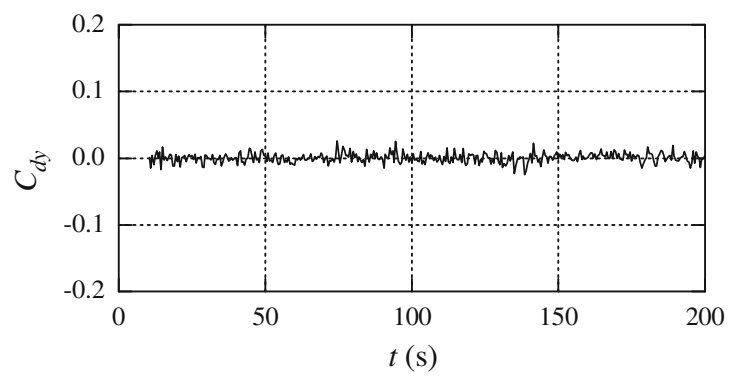

(h)

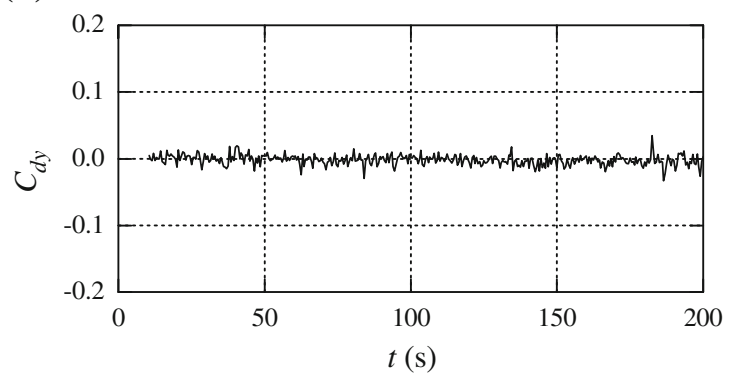

Fig. 4 Effective drag coefficient and corresponding energy spectra for Reynolds numbers $\left(1.0 \times 10^{4}\right.$ to $\left.2.0 \times 10^{5}\right)$. a Wave only, b $K C=0.6$, $R e=10,000, \mathbf{c} K C=0.6, R e=100,000, \mathbf{d} \quad K C=0.6, R e=150,000$, e $K C=0.6, R e=200,000$, f Wave only, $\mathbf{g} K C=0.6, R e=10,000$, h $K C=0.6, R e=100,000, \mathbf{i} K C=0.6, R e=150,000, \mathbf{j} K C=0.6, R e=200,000, \mathbf{k}$ Wave only, $\mathbf{l} K C=0.6, R e=10,000, \mathbf{m} K C=0.6$, $R e=100,000, \mathbf{n} K C=0.6, R e=150,000, \mathbf{o} K C=0.6, R e=200,000$ 
(i)

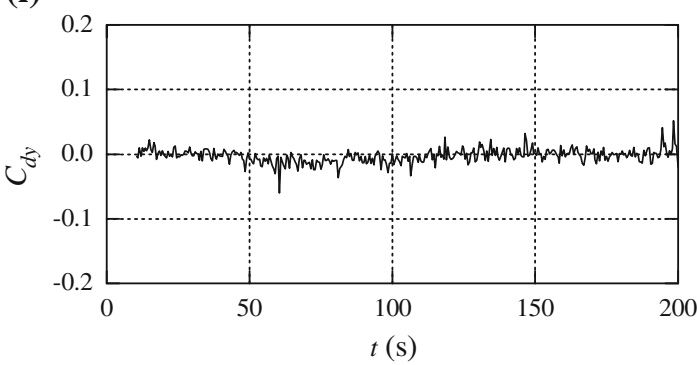

(k)

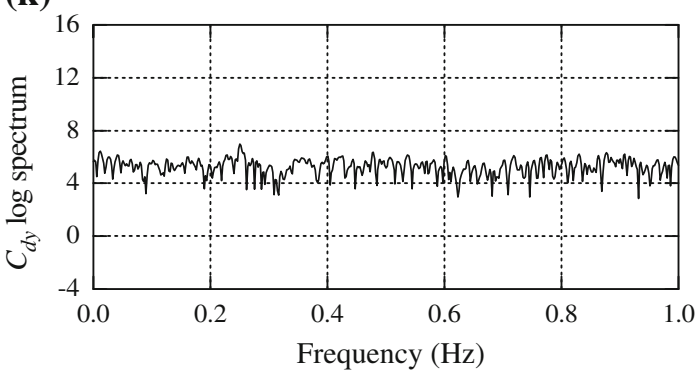

(m)

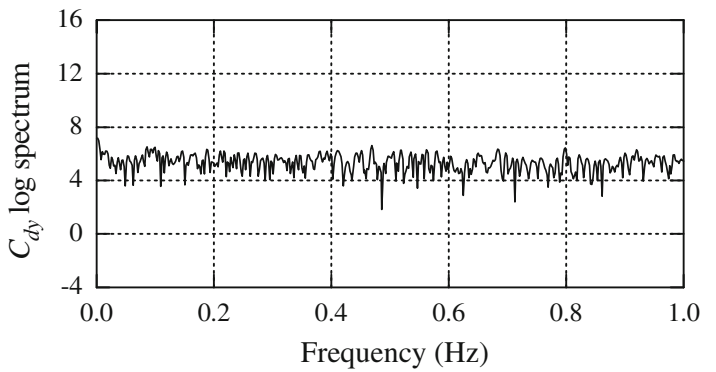

(o)

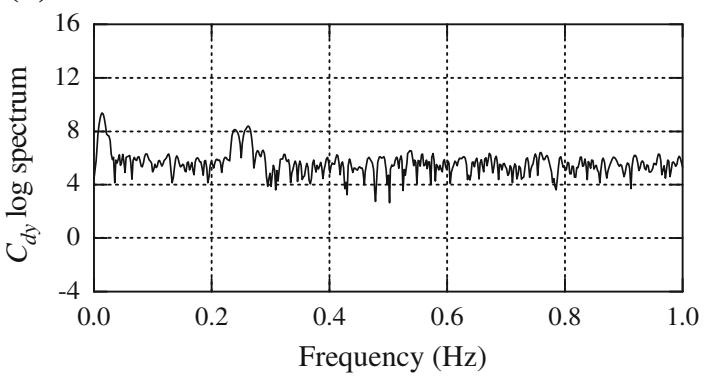

(j)

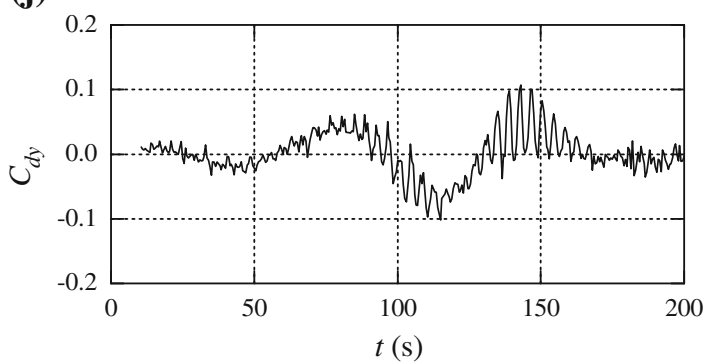

(l)

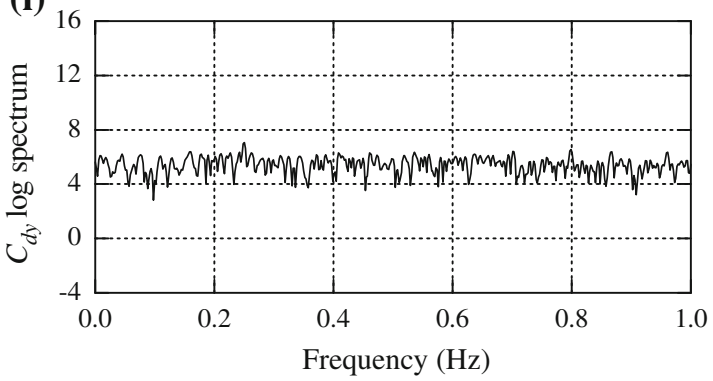

(n)

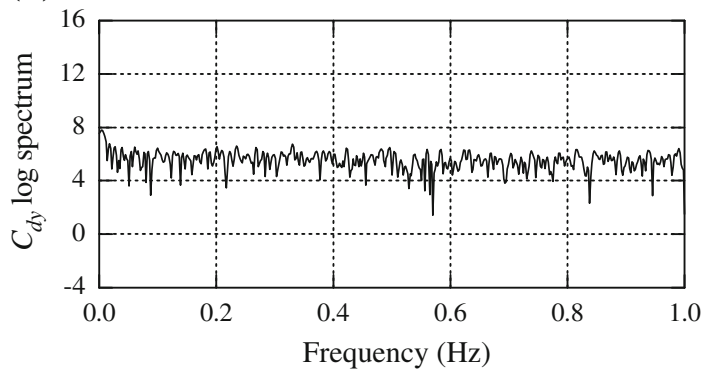

Fig. 4 continued

than $R e_{\text {critical }}=u_{p} L / v=1.5 \times 10^{5}$, the effect of Reynolds number on wave is almost very little. The drag force and vortex form caused by wave-current interaction with $K C=0.6$ as well as weak current are consistent with that of wave action only. Conversely, when the Reynolds number magnitude is the same as $R e_{\text {critical }}$, the wavecurrent nonlinear interaction is obvious. Although with the same $K C$ number, the trailing vortex forms differ from one another. With increasing the Reynolds number from $1.5 \times 10^{5}$ to $6.0 \times 10^{5}$, the vortex form becomes more complicated; however, the separation point remains at the upstream corners at all times for different Reynolds numbers. From Fig. $3 i-j$, it is expected that the vortex feature under wave-current effect becomes much more 

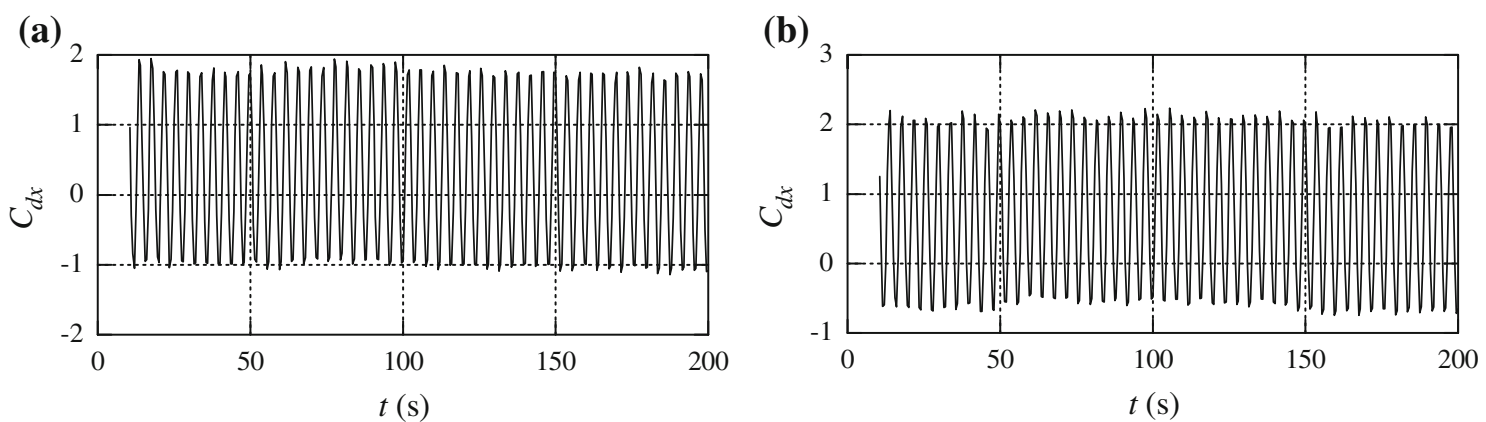

(c)

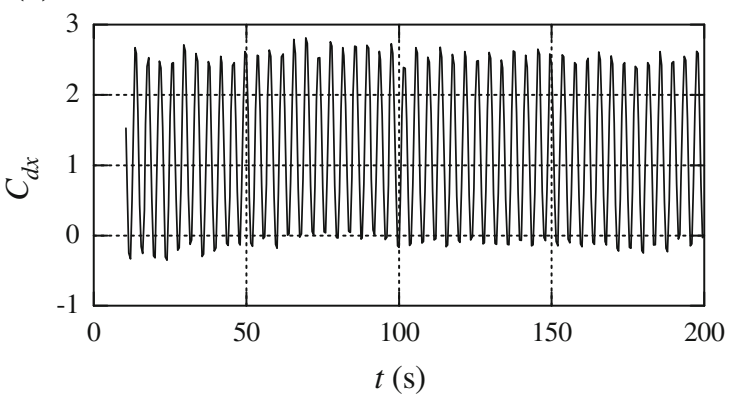

(d)

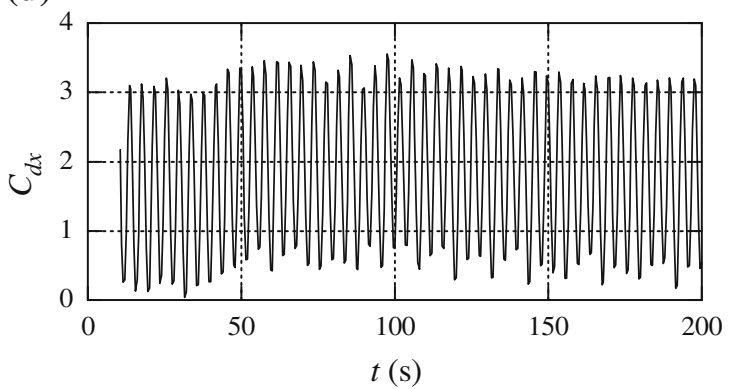

(e)

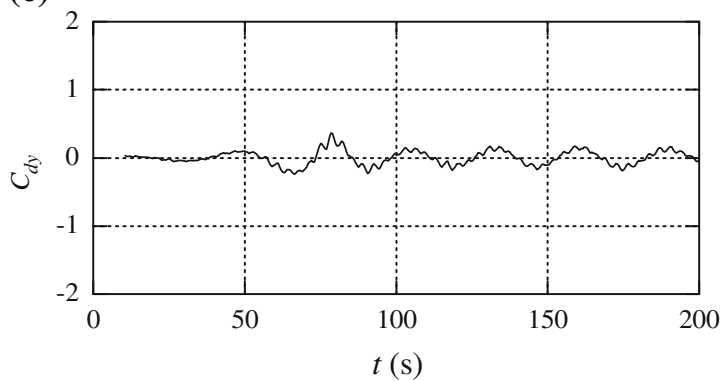

(f)

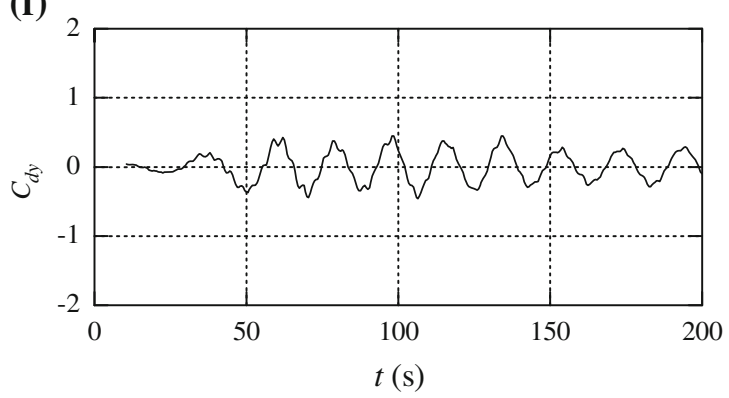

(g)

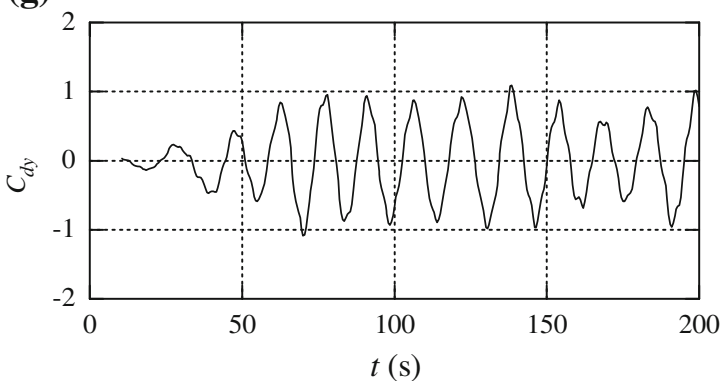

(h)

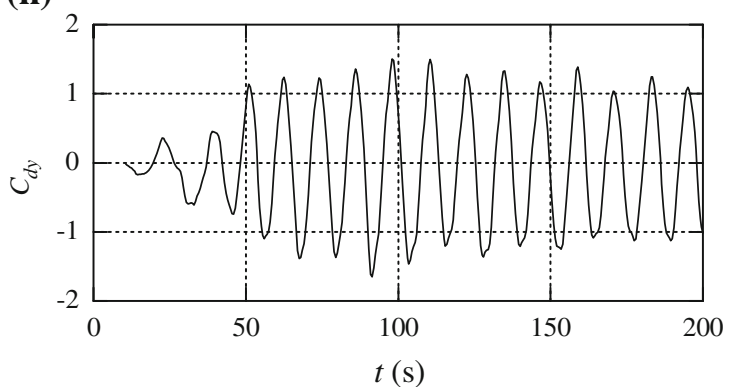

Fig. 5 Effective drag coefficient and corresponding energy spectra for Reynolds numbers $\left(3.0 \times 10^{5}\right.$ to $\left.6.0 \times 10^{5}\right)$. a $K C=0.6, R e=300,000$, b $K C=0.6, R e=400,000$, c $K C=0.6, R e=500,000$, d $K C=0.6, R e=600,000$, e $K C=0.6, R e=300,000$, f $K C=0.6, R e=400,000$, g $K C=0.6, R e=300,000, \mathbf{h} K C=0.6, R e=600,000$ i $K C=0.6, R e=300,000, \mathbf{j} K C=0.6, R e=400,000, \mathbf{k} K C=0.6, R e=500,000$, l $K C=0.6, R e=600,000$

complicated in comparison to that of the current-only effect due to the presence of wave oscillating motion.

In the presence of wave, there also exists inertial force caused by the unsteady oscillating motion besides the drag force. Thus, to simplify the analysis and facilitate the comparisons among different cases, all forces are normalized by $\rho u^{2}(L d) / 2$, in this study $u$ in all cases is taken as $0.6 \mathrm{~m} / \mathrm{s}$ so that the effective force coefficient in the $x$ and $y$ directions, e.g., $C_{\mathrm{d} x}$ and $C_{\mathrm{d} y}$, are obtained. The time history curve of effective drag coefficient and 


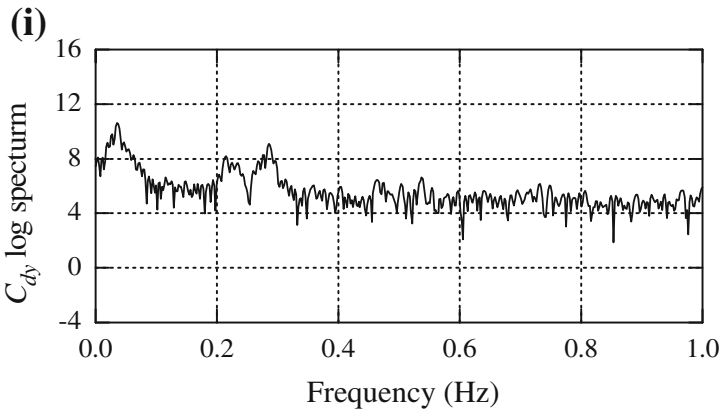

(k)

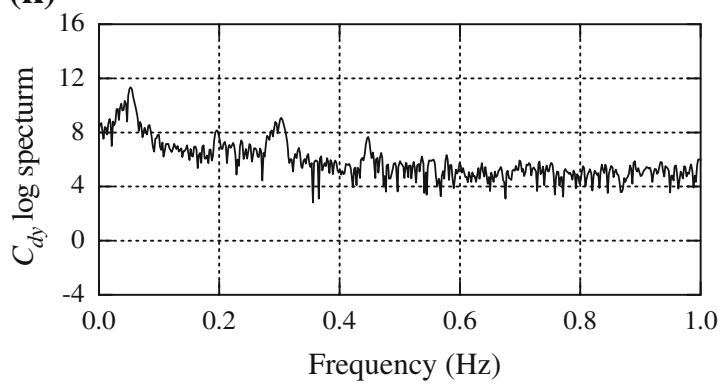

(j)

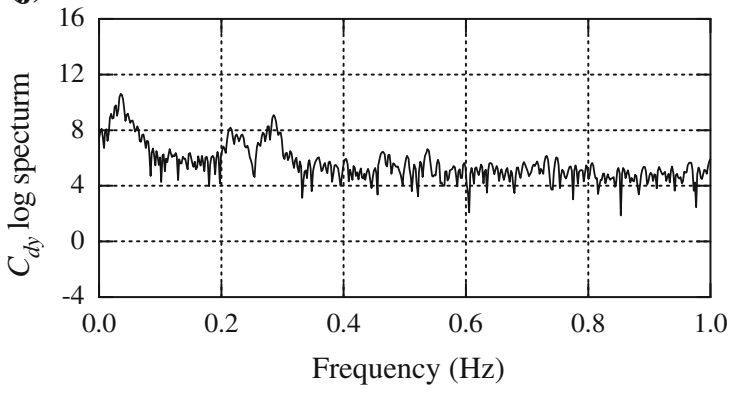

(l)

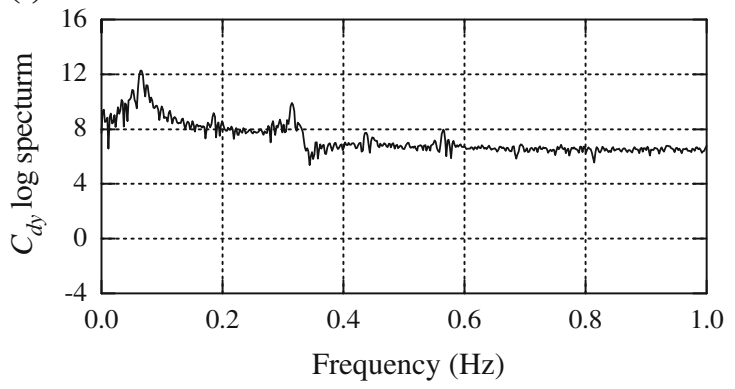

vertical velocity $(W)$ magnitude is increased because of the increasing of Reynolds number. Thus, vertical velocity $W$ should be considered to wave-current interaction with high corresponding Reynolds numbers. It is of great importance to consider the wave-current as three-dimensional flow.

The variations of Strouhal number and effective drag coefficient under different Reynolds numbers are shown in Fig. 7 and Table 2. The vortex shedding frequency and the mean wave-current force are nearly close to zero for the case of Reynolds number smaller than $1.0 \times 10^{5}$, which is the same as the wave-only case. As the Reynolds number increases, the Strouhal number, the mean force coefficient and the RMS of coefficients increase, and they all show the same variation tendency. It is expected that the mean ones, the RMS ones, and Strouhal number are all sensitive to various Reynolds numbers under wave-current effect. From Fig. 7d, it is found that the Strouhal number increases slightly for the higher Reynolds numbers ranging from $3.0 \times 10^{5}$ to $6.0 \times 10^{5}$.

\section{FEM simulation}

Wave-current interaction with a vertical square cylinder is investigated numerically in this study. The results show as follows: 
(a)

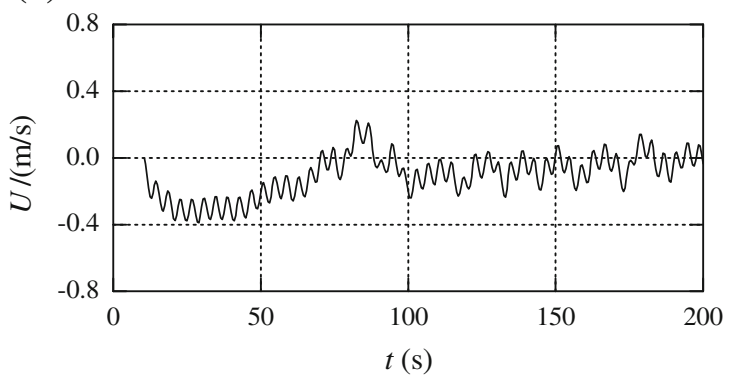

(c)

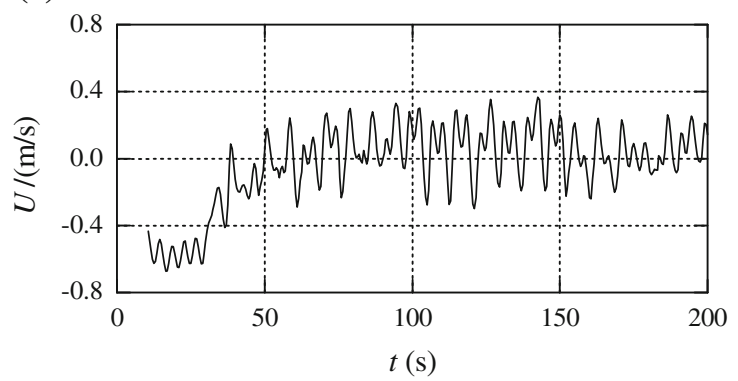

(e)

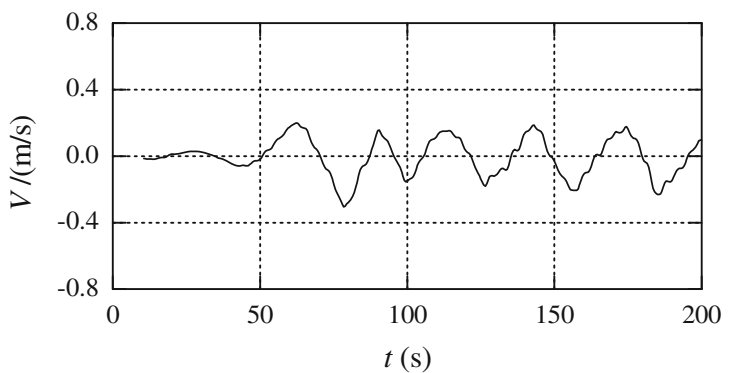

(g)

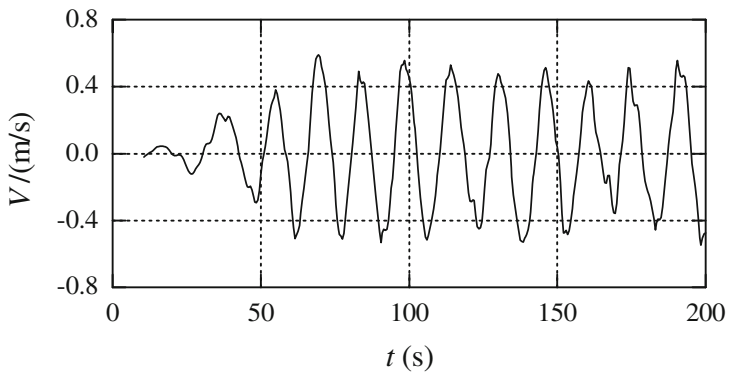

(b)

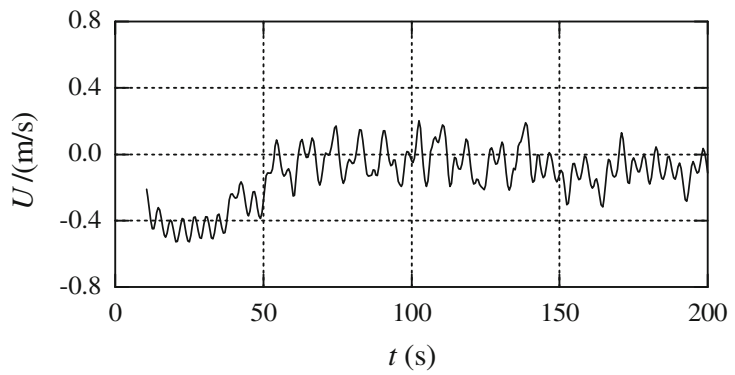

(d)

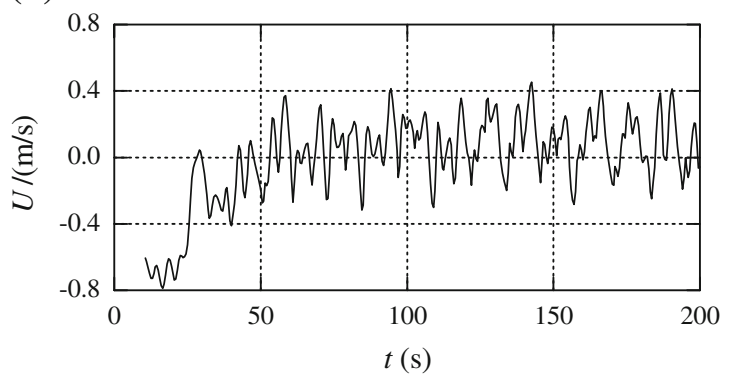

(f)

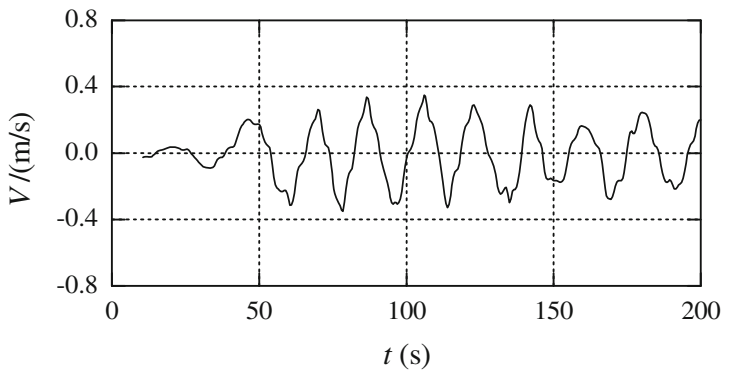

(h)

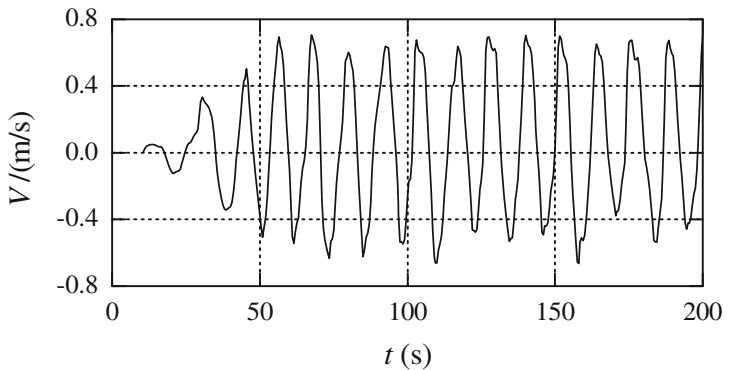

Fig. 6 Velocity in three directions at point $(x=1.5 \mathrm{~m}$ and $y=0.0 \mathrm{~m})$ for different Reynolds numbers. a $K C=0.6, R e=300,000$, b $K C=0.6$, $R e=400,000$, c $K C=0.6, R e=500,000$, d $K C=0.6, R e=600,000$, e $K C=0.6, R e=300,000$, f $K C=0.6, R e=400,000$, g $K C=0.6$, $R e=500,000, \mathbf{h} K C=0.6, R e=600,000 \mathbf{i} K C=0.6, R e=300,000, \mathbf{j} K C=0.6, R e=400,000, \mathbf{k} K C=0.6, R e=500,000, \mathbf{l} K C=0.6$, $R e=600,000$

(1) The vortex shedding frequency has been reduced because of wave-current nonlinear interaction.

(2) When the corresponding Reynolds number is smaller than a critical one, current effect on wave can be nearly neglected.
(3) With the Reynolds number increasing, however, wave-current-structure interaction is sensitive to the Reynolds number; in this case, the effect of Reynolds number on the global quantities, the mean value, 

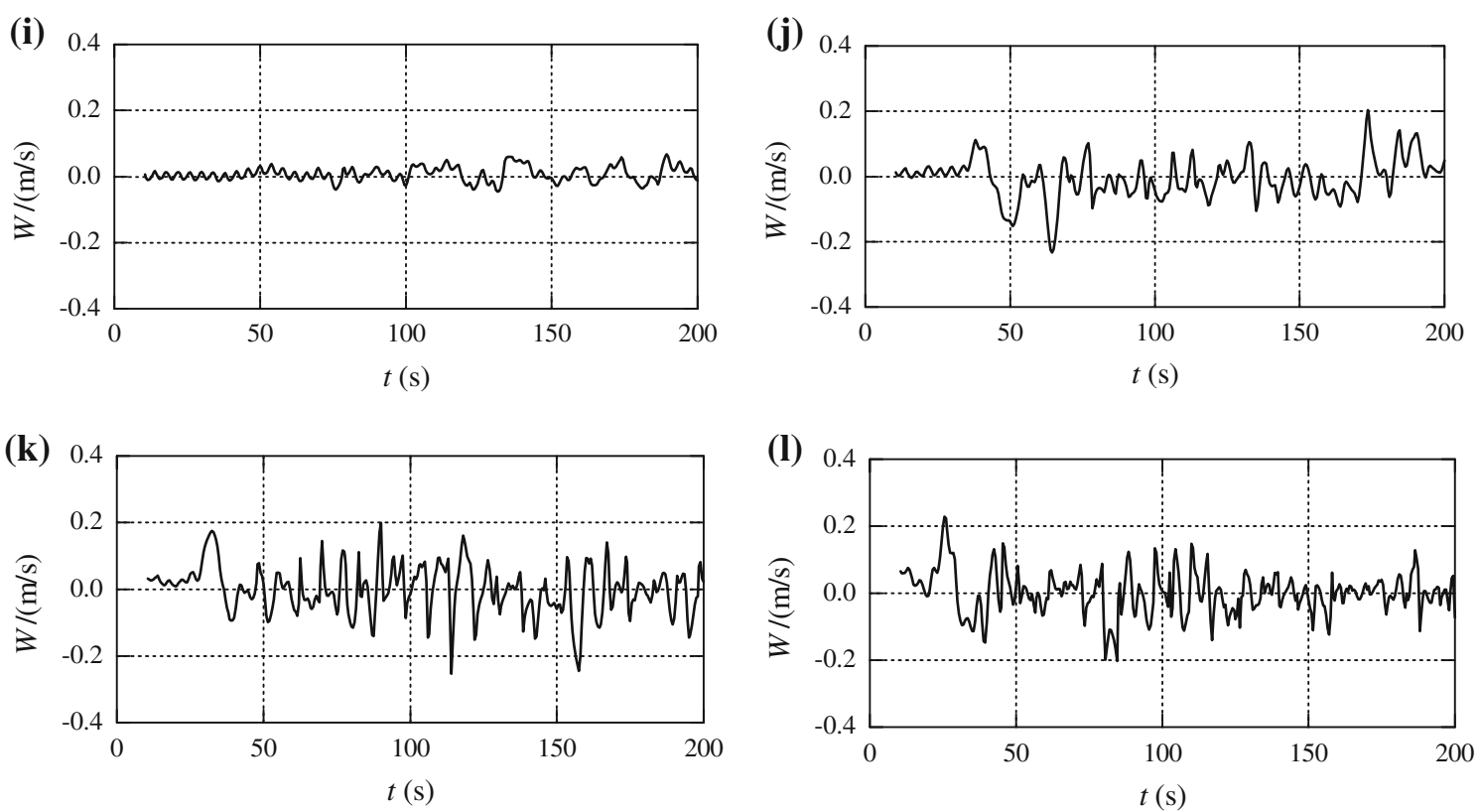

Fig. 6 continued
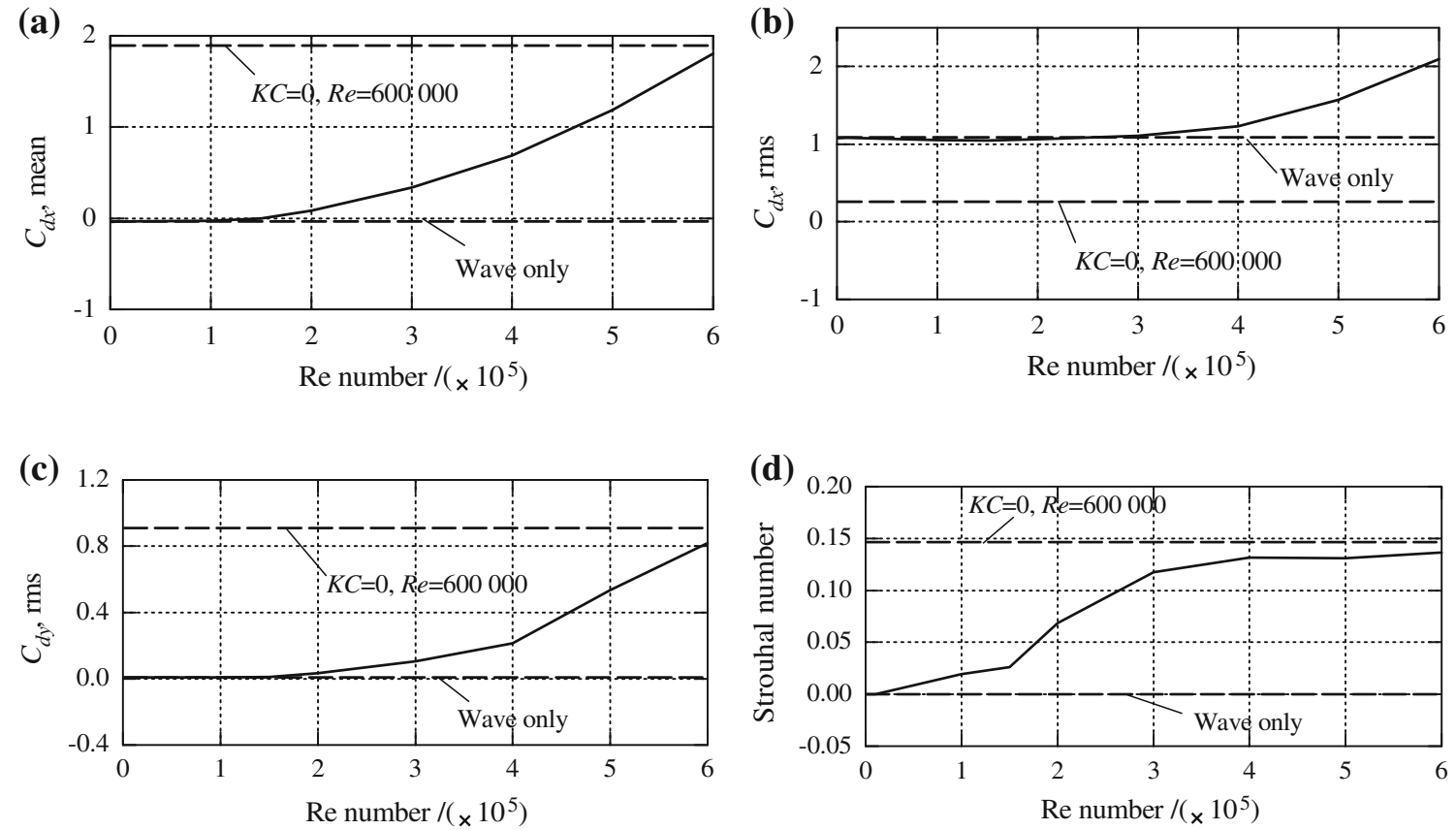

Fig. 7 The mean and RMS drag coefficient as well as Strouhal number (St) versus different Reynolds numbers. a $C_{\mathrm{d} x}$ (mean), b $C_{\mathrm{d} x}$ (RMS), c $C_{\mathrm{d} y}(\mathrm{RMS})$, d St number

RMS value of drag force coefficient and turbulent feature are much more obvious and unignorable.

(4) The vertical velocity is increased with the Reynolds muber increasing, and the magnitude of it weighs against the streamwise velocity. Thus, it is of great importance to consider the wave-current interaction as three-dimensional flow. 
Table 2 Calculated mean force coefficients, RMS of fluctuation of coefficients, and normalized shedding frequencies (Strouhal number St) for various Reynolds numbers

\begin{tabular}{|c|c|c|c|c|}
\hline Wave-current parameters & $\bar{C}_{\mathrm{d} x}$ & $\bar{C}_{\mathrm{d} x}^{\prime}$ & $\bar{C}_{d y}^{\prime}$ & St \\
\hline$K C=0.6, R e=1.0 \times 10^{3}$ & -0.0373 & 1.0877 & 0.0074 & - \\
\hline$K C=0.6, R e=1.0 \times 10^{4}$ & -0.0374 & 1.0803 & 0.0075 & - \\
\hline$K C=0.6, R e=1.0 \times 10^{5}$ & -0.0349 & 1.0815 & 0.0084 & - \\
\hline$K C=0.6, R e=1.0 \times 10^{5}$ & -0.0259 & 1.0551 & 0.0081 & 0.019531 \\
\hline$K C=0.6, R e=1.0 \times 10^{5}$ & -0.0014 & 1.0505 & 0.0111 & 0.026042 \\
\hline$K C=0.6, R e=1.0 \times 10^{5}$ & 0.0849 & 1.062 & 0.0336 & 0.0685 \\
\hline$K C=0.6, R e=1.0 \times 10^{5}$ & 0.338 & 1.1086 & 0.1058 & 0.117333 \\
\hline$K C=0.6, R e=1.0 \times 10^{5}$ & 0.687 & 1.2319 & 0.2145 & 0.13175 \\
\hline$K C=0.6, R e=1.0 \times 10^{5}$ & 1.1892 & 1.5709 & 0.5352 & 0.131 \\
\hline$K C=0.0, R e=1.0 \times 10^{5}$ & 1.8927 & 0.2568 & 0.9080 & 0.1465 \\
\hline
\end{tabular}

Acknowledgments The research is supported by the National Natural Science Foundation of China (No. 51178397), and Technological Research and Development Programs of the Ministry of Railways (No. 2010G004-L).

Open Access This article is distributed under the terms of the Creative Commons Attribution License which permits any use, distribution, and reproduction in any medium, provided the original author(s) and the source are credited.

\section{References}

1. Park JC, Kim MH, Miyata H (2001) Three-dimensional numerical wave tank simulations on fully nonlinear wave-current-body interactions. J Mar Sci Technol 6(2):70-82

2. Deardorff JW (1970) A numerical study of three-dimensional turbulent channel flow at large Reynolds numbers. J Fluid Mech 41(2):453-480

3. Sohankar A (2006) Flow over a bluff body from moderate to high Reynolds numbers using large eddy simulation. Comput Fluids 35(10):1154-1168

4. Koo W, Kim MH (2007) Current effects on nonlinear wave-body interactions by a 2D fully nonlinear numerical wave tank. J Waterw Port Coast Ocean Eng 133(2):136-146

5. Li Y, Lin M (2010) Hydrodynamic coefficients induced by waves and currents for submerged circular cylinder. Procedia Eng 4:253-261

6. Cheng M, Whyte DS, Lou J (2007) Numerical simulation of flow around a square cylinder in uniform-shear flow. J Fluids Struct 23(2):207-226

7. Vengadesan S, Nakayama A (2005) Evaluation of LES models for flow over bluff body from engineering application perspective. Sadhana 30(1):11-20
8. Li CW, Lin P (2001) A numerical study of three-dimensional wave interaction with a square cylinder. Ocean Eng 28(12): $1545-1555$

9. Lin P (2004) A numerical study of solitary wave interaction with rectangular obstacles, Coast Eng 51(1):35-51

10. Lin P, Li CW (2003) Wave-current interaction with a vertical square cylinder. Ocean Eng 30(7):855-876

11. Lin P (2006) A multiple-layer sigma-coordinate model for simulation of wave-structure interaction. Comput Fluids 35(2): $147-167$

12. Tan CJ, Zhu B (2010) Numerical study of three-dimensional wave-current interaction with cylinders. Chin J Appl Mech 27(4):680-686

13. Tan CJ (2009) Coupled cable-deck vibration research of long span cable stayed bridge under wave effect. Southwest Jiaotong University of China, Chengdu

14. Smagorinsky JS (1963) General circulation experiments with the primitive equations, part I: the basic experiment. Mon Weather Rev 91:99-163

15. Lin PZ, Li CW (2002) A $\sigma$-coordinate three-dimensional numerical model for free surface wave propagation. Int J Numer Methods Fluids 38(11):1045-1068

16. Benque JP (1982) A new method for tidal current computation. J Waterw Port Coast Ocean Div 108(3):396-417

17. Lyn DA (1989) Phase-averaged turbulence measurements in the separated shear flow around square cylinder, In: Proceedings of the 23rd congress of International Association for Hydraulic Research, Ottawa, pp A85-A92

18. Vickery BJ (1966) Fluctuating lift and drag on a long cylinder of square cross-section in a smooth and in a turbulent stream. J Fluid Mech 25(3):481-494

19. Lee BE (1975) The effect of turbulence on the surface pressure field of a square prism. J Fluid Mech 69(2):263-282 